\title{
The management and clinical knowledge of headache disorders among general practitioners in Norway: a questionnaire survey
}

Espen Saxhaug Kristoffersen ${ }^{1,2,3^{*}}$ (D), Kashif Waqar Faiz ${ }^{2}$, Jakob Møller Hansen ${ }^{4}$, Erling Andreas Tronvik ${ }^{5,6}$, Jan C. Frich? ${ }^{7}$ Christofer Lundqvist ${ }^{2,8,9}$ and Bendik Slagsvold Winsvold ${ }^{3,10}$

\begin{abstract}
Background: General practitioners (GPs) diagnose and manage a majority of headache patients seeking health care. With the aim to understand the potential for clinical improvement and educational needs, we performed a study to investigate Norwegian GPs knowledge about headache and its clinical management.

Methods: We invited GPs from a random sample of 130 Norwegian continuous medical education (CME) groups to respond to an anonymous questionnaire survey.

Results: 367 GPs responded to the survey (73\% of invited CME groups, 7.6\% of all GPs in Norway). Mean age was 46 (SD 11) years, with an average of 18 (SD 10) years of clinical experience. In general the national treatment recommendations were followed, while the International Classification of Headache Disorders and other international guidelines were rarely used. Overall, $80 \%(n=292)$ of the GPs suggested adequate prophylactic medication for frequent episodic migraine, while $28 \%(n=101)$ suggested adequate prophylactic medication for chronic tension-type headache $(\mathrm{CTTH})$. Half $(52 \%, n=191))$ of the respondents were aware that different types of acute headache medication can lead to medication-overuse headache $(\mathrm{MOH})$, and 59\% $(n=217)$ knew that prophylactic headache medication does not lead to $\mathrm{MOH}$. GPs often used MRI in the diagnostic work-up. GPs reported that lack of good treatment options was a main barrier to more optimized treatment of headache patients.
\end{abstract}

Conclusion: The knowledge of management of $\mathrm{CTTH}$ and $\mathrm{MOH}$ was moderate compared to migraine among Norwegian GPs.

Keywords: Migraine, Medication-overuse headache, Education, Quality, Guidelines, Primary care

* Correspondence: e.s.kristoffersen@medisin.uio.no

${ }^{1}$ Department of General Practice, University of Oslo, PO Box 1130, Blindern,

0318 Oslo, Norway

Full list of author information is available at the end of the article

(c) The Author(s) 2021, corrected publication 2023. Open Access This article is licensed under a Creative Commons Attribution 4.0 International License, which permits use, sharing, adaptation, distribution and reproduction in any medium or format, as long as you give appropriate credit to the original author(s) and the source, provide a link to the Creative Commons licence, and indicate if changes were made. The images or other third party material in this article are included in the article's Creative Commons licence, unless indicated otherwise in a credit line to the material. If material is not included in the article's Creative Commons licence and your intended use is not permitted by statutory regulation or exceeds the permitted use, you will need to obtain permission directly from the copyright holder. To view a copy of this licence, visit http://creativecommons.org/ licenses/by/4.0/. The Creative Commons Public Domain Dedication waiver (http://creativecommons.org/publicdomain/zero/1. 0/) applies to the data made available in this article, unless otherwise stated in a credit line to the data. 


\section{Background}

The focus on headache in the curriculum at medical schools and in specialist training throughout the world is limited [1-3]. Insufficient education, training and knowledge about headache may be a cause of diagnostic failure, inappropriate treatment, and low patient satisfaction among headache patients [4]. Headache is one of the main reasons why patients contact a general practitioner (GP), and because most people with headaches are well-managed by the GPs, only a small proportion of patients are referred to more specialized care [5-8]. GPs play a key role both in treatment and as gatekeepers for referrals to specialist care [9]. However, for this to function, it is imperative that the GP has sufficient knowledge and validated tools for everyday use. The burden of headache and migraine is high and the findings suggest that diagnosis and management in the health care system is still limited [10-12]. International guidelines and National recommendations for diagnosis and management developed by the National Advisory Unit on Headache in Norway exist [12-15], but data is very limited on how GPs actually experience and manage headache patients [16, 17]. In the present exploratory descriptive study we investigate the knowledge among Norwegian GPs about headache and the clinical management of patients with headache.

\section{Methods}

\section{Design and setting}

Primary health care in Norway are provided by GPs in a patient-list system. More than $99.8 \%$ of people living in Norway (5.4 million) use the GP patient-list system [18]. The average patient list consists of approximately 1100 patients per GP. Norwegian GPs are on average 47.2 years old, $45.8 \%$ are women and $63.2 \%$ are certified specialists in general practice [18].

In 2017 it became mandatory for all GPs in Norway to be a certified GP specialist or under training to become a certified GP specialist. At the time of this survey the training program to become a certified GP specialist includes 1.5 years of internship (usually internal medicine, general surgery, and general practice), followed by 4 years of GP training, of which 1 year must be spent in another clinical specialty. In addition, clinical training courses and individual supervision must be completed; however, a headache course is optional. GP specialists must also participate in mandatory peer continuous medical education (CME) groups to be re-certified every fifth year. In everyday clinical practice the responsibilities and working situation does not differ between GPs under training and those certified as GP specialists.

We invited GPs to respond to an anonymous questionnaire survey between 2018 and 2019 through invitations to a sample of 130 Norwegian CME groups. The
CME groups were invited based on a representative geographical distribution with both rural and urban GPs. The CME groups were invited by email and received one reminder. The administrator (one of the GPs) of each CME group distributed the invitation to the others. CME groups usually consists of 3-5 GPs, but there exists no updated list for all GPs in CME groups in Norway. Participants were required to complete questionnaires individually in a web-based questionnaire.

\section{Questionnaire}

We developed a questionnaire (supplement 1) based on available literature and the authors' experience in health service research, general practice and headache management [3, 17]. The questionnaire covered background variables and the participants responded to a short multiple-choice and open questions about knowledge of and experience in the management of headache, use of the national treatment recommendations and the International Classification of Headache Disorders 3rd edition (ICHD-3) and barriers to optimised treatment.

We used a 3-item scale with the options "good", "moderate" and "poor" to assess self-rated knowledge of migraine, tension-type headache, medication-overuse headache $(\mathrm{MOH})$, and cluster headache/trigeminal autonomic cephalalgias (TACs).

We asked GPs how often they used headache diaries, imaging, web-sites (BMJ Best Practice and UpToDate are both available for free for clinicians in Norway), national treatment recommendations, and the ICHD-3, with the response options i) every time, ii) two out of three times, iii) half the time, iv) one out of three times/ rarely, and v) never. The categories were re-classified into " $\geq 2 / 3$ ", "half the time", and " $\leq 1 / 3$ ", due to few responses in the categories every time and never.

Two different headache cases were used to assess the participants' suggestions for medical treatment of patients with i) chronic tension-type headache (CTTH), and ii) frequent episodic migraine (1-2 migraine attacks every week), two of the most seen headache types in general practice. Several questions concerned $\mathrm{MOH}$.

To assess the main barriers to optimised management of patients with headache and reasons for referral, we asked the participants to rank pre-specified statements.

\section{Outcomes}

Outcomes were mainly categorised descriptive data based on the described variables. In addition, demographic, practice-related and medical training-related predictors for pre-defined logistic answers (yes/no) regarding knowledge of acute and prophylactic medical treatment of CTTH, migraine and $\mathrm{MOH}$ for the following outcomes were analysed: 
Knowledge of CTTH prophylaxis was defined as yes if the participant suggested a tri-cyclic antidepressant as the prophylactic medication of choice in CTTH.

Knowledge of migraine prophylaxis was defined as yes if the participant suggested a beta-blocker, lisinopril, candesartan, topiramate, amitriptyline, valproate or Botulinum Toxin A as the prophylactic medication of choice in frequent episodic migraine.

Knowledge of prophylactic medications and $\mathrm{MOH}$ was defined as yes if the participant answered correctly that commonly used prophylactics (antiepileptic drugs, antihypertensive drugs, antidepressant drugs, and botulinum toxin A) do not induce $\mathrm{MOH}$.

Knowledge of acute medications and $\mathrm{MOH}$ was defined as yes if the participant answered correct that simple analgesics, combination analgesics, opioids and triptans may induce $\mathrm{MOH}$. As ergotamine is very rarely used in Norway, this medication group was not included.

\section{Statistical analyses}

For descriptive data, proportions, means, and standard deviations (SD), or 95\% confidence intervals (CI) are given. Groups were compared using the $t$-test (continuous data) or the $\chi^{2}$ test (categorical data).

We used multiple logistic regression analysis to evaluate the effect of age, gender, certified specialist in general practice, number of patients on the GP list, and whether the GP had attended a headache course on the outcomes (yes/no) pertaining to knowledge of adequate prophylactic medication use for CTTH and frequent episodic migraine as well as prophylactic and acute medication use in $\mathrm{MOH}$.

The results of the questions of barriers to optimal management and referrals are presented descriptively as proportions, medians and interquartile range (IQR). Comparisons between the ranked statements were done by Wilcoxon Matched-Pairs Signed Ranks test.

Statistical significance was defined by $p<0.05$, using a two-sided test. As this was an exploratory descriptive study, we did not perform an a priori power calculation or adjust for multiple comparisons. Statistical analyses were performed using IBM SPSS Statistics, Version 27.00 (SPSS Inc., Chicago, IL, USA).

\section{Results}

In total, 367 GPs responded to the survey. Respondents were recruited from 95 (73\%) out of 130 invited CME groups. Among the CME groups that did not participate, $26(20 \%)$ did not respond to the invitation, and nine (7\%) declined to take part in the study. The mean age of respondents was 46 (range 25-70) years, half (49\%) of the respondents were women, and $71 \%$ were certified specialists. On average, the respondents had almost 15
Table 1 Descriptive data of the participants $(N=367)$

\begin{tabular}{ll}
\hline Sex $\mathbf{n}(\%)$ & \\
\hline Women & $179(49)$ \\
Men & $188(51)$ \\
Age, mean (SD) & $46.0(10.5)$ \\
Years as physician, mean (SD) & $17.8(10.4)$ \\
Years as general practitioner, mean (SD) & $14.4(10.3)$ \\
Certified specialist in general practice, n (\%) & \\
$\quad$ Yes & $262(71)$ \\
No & $105(29)$ \\
Years as certified specialist in general practice, mean & $12.3(9.2)$ \\
(SD) & $1133(400-$ \\
Number of patients on list, mean (min-max) & $2200)$ \\
In-person consultations per day, mean (SD) & $18.7(3.8)$ \\
Attended headache course, $n$ (\%) & \\
Yes & $83(23)$ \\
No & $284(77)$ \\
\hline
\end{tabular}

years of experience in general practice. Table 1 presents descriptive data about the respondents.

\section{Knowledge}

Knowledge of which medications may lead to $\mathrm{MOH}$ is reported in Fig. 1. Fifty-nine percent $(n=217)$ answered all questions on $\mathrm{MOH}$ (i.e. acute medications and prophylaxis) correctly, but many wrongly stated that one of the most commonly used headache prophylactics could lead to $\mathrm{MOH}(17 \%(n=63)$ for anti-hypertensives, $28 \%(n=101)$ for anti-epileptics, and 22\% $(n=79)$ for antidepressant drugs). Fifty-nine\% $(\mathrm{n}=217)$ knew that none of the prophylactic headache medication induce $\mathrm{MOH}$. More than $90 \%(n=332)$ of the respondents

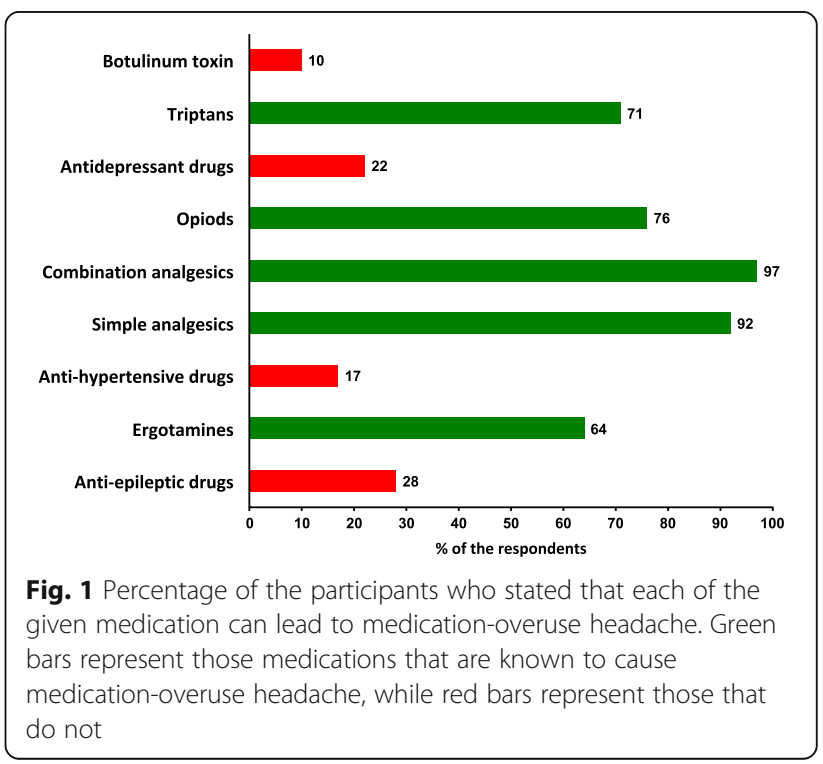


knew that simple analgesics and combination analgesics may lead to $\mathrm{MOH}$. Overall, $52 \%(n=191)$ of the respondents answered all questions on acute headache medication correct i.e. simple analgesics, combination analgesics, opioids and triptans may induce $\mathrm{MOH}$. Furthermore, $24 \%(n=87)$ wrongly stated that highly potent opioids, and $29 \%(n=106)$ that triptans, cannot lead to $\mathrm{MOH}$. A significantly larger proportion of GP specialists compared to non-specialists answered all questions about medication and $\mathrm{MOH}$ correctly $(64 \%(n=167)$ vs. $48 \%(n=50), p=0.005)$.

Figure 2 shows that self-reported knowledge was reported as good concerning migraine and tension-type headache in two out of three GPs, whereas few GPs stated that they had good general knowledge about cluster headache, TACs and $\mathrm{MOH}$. There were no gender differences in self-reported knowledge, but specialists reported better knowledge about migraine, cluster headache, TACs and $\mathrm{MOH}$ than non-specialists $(p<0.01)$.

A higher proportion of those with good self-reported knowledge of migraine answered the questions about prophylactic treatment of migraine correct compared to those with poor self-reported knowledge ( $80 \%$ vs $33 \%$, $p=0.043)$. There was a non-significant difference in the self-reported knowledge of TTH and the reported correct use of preventives in CTTH. Correct use was reported by $32 \%$ (good self-reported knowledge), $22 \%$ (medium self-reported knowledge) and $0 \%$ (poor selfreported knowledge), $p=0.06$ for TTH. There was a significant difference in the self-reported knowledge of $\mathrm{MOH}$ and the actual knowledge about acute medication overuse and use of preventives in $\mathrm{MOH}$. Adequate knowledge of $\mathrm{MOH}$ was found in $72 \%$ (good selfreported knowledge), 57\% (medium self-reported knowledge) and 51\% (poor self-reported knowledge), $p=$ 0.033. Altogether, the proportion that self-reported good knowledge about migraine, TTH and $\mathrm{MOH}$ and answered the questions about these disorders satisfactorily were $80 \%, 32 \%$ and $72 \%$, respectively.

In the logistic regression models, none of the tested variables were associated with a higher proportion of respondents with knowledge about prophylactic headache medication $(\mathrm{CTTH}$, migraine and $\mathrm{MOH})$. The only significant finding was that GP specialists had an increased odds of 1.9 (95\% CI 1.1; 3.3, $p=0.02$ ) for knowing that acute headache medication may induce $\mathrm{MOH}$.

\section{Clinical diagnostics and management}

The most frequent response to the question "Do you find headache to be a clinically difficult professional challenge (1=difficult and $4=$ =easy)" was alternative 2 (50\%), followed by alternative $3(41 \%)$. A majority of $59 \%(n=215)$ used the national treatment recommendations for headache in $\geq 2 / 3$ of consultations. Other international resources such as ICHD-3, BMJ Best Practice, UpToDate or Google search were used rarely (Table 2). There were no differences between specialists and nonspecialists.

Headache diaries were used regularly (i.e. in $\geq 2 / 3$ of consultations) by $35 \%(n=127)$ for diagnostic purposes and by $30 \%(n=110)$ for follow-up. Thirty-four percent reported that they received the diary back from the patients in $2 / 3$ of follow-ups. There were no significant differences in the use of diaries between specialists and non-specialists.

Thirty-three percent $(n=123)$ of participants responded that they asked patients about disability, social functioning, and sick leave in every consultation, and an additional 27\% $(n=99)$ asked about these factors in $>2 / 3$ consultations. Seventeen percent $(n=63)$ asked these questions in $1 / 3$ of consultations or fewer.

Table 3 shows participants' reported use of imaging. Almost all GPs used imaging for headaches with focal neurological symptoms, and $84 \%$ used imaging if the headache was not responding to treatment. Sixty-two

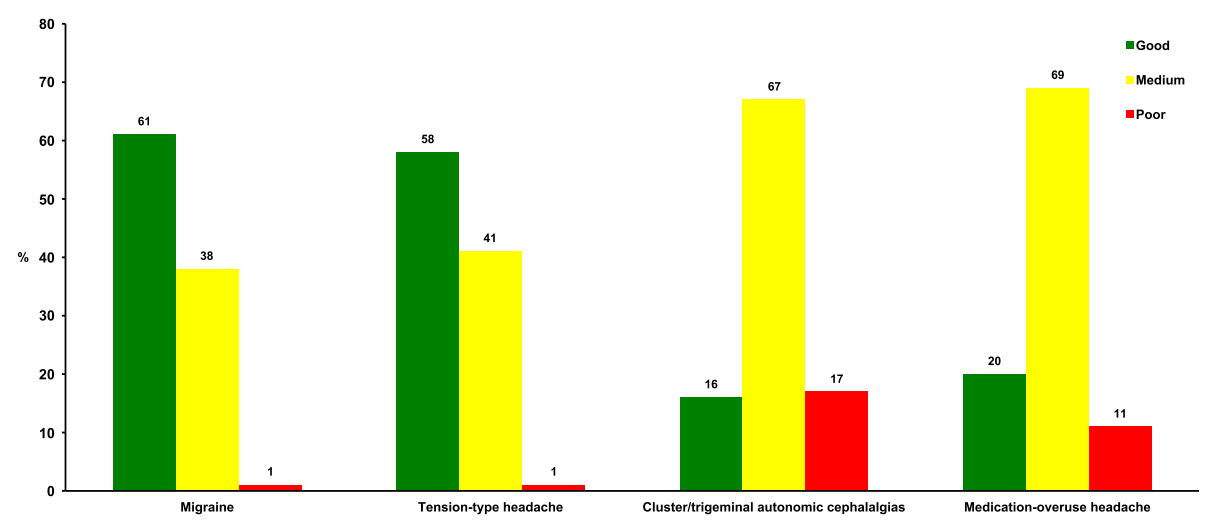

Fig. 2 Percentage of the participants responding good (green), medium (yellow), or poor (red) to the question "How do you rate your own knowledge of migraine, tension-type headache, cluster headache/trigeminal autonomic cephalalgias, and medication-overuse headache?" 
Table 2 General Practitioners' use of diagnostic and treatment tools for diagnosis and follow-up of headache patients. All figures are numbers (\%)

\begin{tabular}{|c|c|}
\hline & All $(\mathrm{N}=367)$ \\
\hline \multicolumn{2}{|c|}{ Headache diary for diagnosis } \\
\hline$\geq 2 / 3$ times & $127(35)$ \\
\hline $1 / 2$ times & $93(25)$ \\
\hline$\leq 1 / 3$ times & $147(40)$ \\
\hline \multicolumn{2}{|c|}{ Headache diary for follow-up } \\
\hline$\geq 2 / 3$ times & $111(30)$ \\
\hline $1 / 2$ times & $77(21)$ \\
\hline$\leq 1 / 3$ times & $179(49)$ \\
\hline \multicolumn{2}{|c|}{ National treatment recommendations } \\
\hline$\geq 2 / 3$ times & $215(59)$ \\
\hline $1 / 2$ times & $65(18)$ \\
\hline$\leq 1 / 3$ times & $87(24)$ \\
\hline \multicolumn{2}{|c|}{ International Classification of Headache Disorders } \\
\hline$\geq 2 / 3$ times & $30(8)$ \\
\hline $1 / 2$ times & $11(3)$ \\
\hline$\leq 1 / 3$ times & $326(89)$ \\
\hline \multicolumn{2}{|c|}{ BMJ Best Practice and/or UpToDate } \\
\hline$\geq 2 / 3$ times & $9(3)$ \\
\hline $1 / 2$ times & $11(3)$ \\
\hline$\leq 1 / 3$ times & $347(95)$ \\
\hline \multicolumn{2}{|l|}{ Google search } \\
\hline$\geq 2 / 3$ times & $7(2)$ \\
\hline $1 / 2$ times & $15(4)$ \\
\hline$\leq 1 / 3$ times & $345(94)$ \\
\hline
\end{tabular}

percent of participants reported using imaging if the patient had concerns and anxiety about brain tumor or other intracranial pathology. Overall, GPs rated MRI as more useful than CT, 59\% found MRI useful in headache diagnostics in at least half of the patients, while the corresponding number for CT was $21 \%$.

Figure 3 show the proportion of respondents who would treat patients with CTTH and frequent episodic migraine (1-2 migraine attacks every week) with acute headache medication prophylactic headache medication, and physiotherapy, respectively. Sixty-nine percent of respondents would treat a patient with CTTH with acute medication, with significantly fewer GP specialists than non-specialists $(65 \%$ vs. $80 \%, p=0.004)$. Simple analgesics (paracetamol or a combination of paracetamol and ibuprofen/other NSAIDs) were the most commonly suggested acute medications for CTTH. Among the 42\% $(n=154)$ that suggested prophylactic medication for CTTH, amitriptyline was suggested by $65 \%(n=100)$, but as many as $17 \%(n=26)$ suggested acute headache medications also for prophylactic use (paracetamol/ ibuprofen/other NSAIDs). Overall, 28\% $(n=101)$ of the GPs suggested adequate prophylactic medication according to national recommendations for CTTH.

Triptans $(87 \%, n=319)$, ibuprofen/NSAIDs/acetylsalicylic acid $(19 \%, n=67)$, and paracetamol $(8 \%, n=30)$ were suggested for the acute treatment of frequent migraine. Prophylactic medication was suggested for frequent migraine by $87 \%(n=318)$, with no differences between GP specialists and non-specialists. Candesartan $(60 \%, n=192)$ and beta-blockers $(56 \%, n=179)$ were the most commonly suggested prophylactic medications, while only $4 \%(n=13)$ suggested topiramate. Overall, $80 \%(n=292)$ of the GPs suggested adequate prophylactic medication according to guidelines for frequent episodic migraine.

Seventy percent $(n=254)$ of the respondents reported that they believed that non-prescription drugs were the most commonly used medication by their chronic headache patients to treat headache. Only $4 \%(n=14)$ believed that their chronic headache patients used pain killers with addictive potential. The majority of GPs $(54 \%, n=185)$ thought that a minority $(\leq 40 \%)$ of their chronic headache patients had medication overuse, while $32 \%(n=117)$ of the GPs estimated this to be the case for $40-60 \%$ of their chronic headache patients.

Sixty percent $(n=219)$ reported MOH to be a clinical challenge among their headache patients with significantly more GP specialists than non-specialists finding $\mathrm{MOH}$ a clinical challenge (64\% vs. $50 \%, p=0.009)$. Ninety-seven percent $(n=356)$ used withdrawal as part of their treatment of MOH. Fifty-five percent $(n=200)$ recommended initial withdrawal only, whereas $43 \%$ ( $n=$ 156) used withdrawal combined with initial prophylactic medication. Fifty percent $(n=182)$ proposed sick-leave during the withdrawal phase and $17 \%(n=62)$ recommended rescue medication as a part of the withdrawal strategy. Furthermore, 15\% $(n=55)$ recommended inpatient withdrawal for patients with $\mathrm{MOH}$ (specialists vs. non-specialists $19 \%$ vs. $6 \%, p=0.002$ ).

The GPs answered the question "What do you believe are the main barriers to optimised treatment and management of your headache patients?" (1-6, where 1 is the most important and 6 is the least important barrier) (Fig. 4). Twenty-seven percent $(n=96)$ scored "No good treatment options for many patients" as the main barrier to more optimized treatment of headache patients (median 2, IQR $1-3, p<0.001$ compared to all other barriers). The next two most reported barriers were "Headache patients are difficult and demanding" (median 3, IQR 2-3) and "Too little time in general practice" (median 3 IQR $2-4)$. Only 9\% $(n=33)$ regarded their own insufficient knowledge to be the most important barrier. Lack of financial incentives to treat headache patients was perceived as the least important barrier. 
Table 3 The use of imaging of headache patients among general practitioners. All figures are numbers (\%)

\begin{tabular}{|c|c|}
\hline & All $(\mathrm{N}=367)$ \\
\hline \multicolumn{2}{|c|}{ Do you usually use CT/MRI for the following? } \\
\hline \multicolumn{2}{|c|}{ All new-onset headache (not acute) } \\
\hline Yes & $23(6)$ \\
\hline No & $344(94)$ \\
\hline \multicolumn{2}{|c|}{ Headache not responding to treatment } \\
\hline Yes & $308(84)$ \\
\hline No & $59(16)$ \\
\hline \multicolumn{2}{|c|}{ Headache with focal neurological symptoms } \\
\hline Yes & $362(99)$ \\
\hline No & $5(1)$ \\
\hline \multicolumn{2}{|c|}{ Worsening of a pre-existent headache } \\
\hline Yes & $236(64)$ \\
\hline No & $131(36)$ \\
\hline \multicolumn{2}{|c|}{ If patients have concerns and anxiety about brain tumor or other intracranial pathology } \\
\hline Yes & $228(62)$ \\
\hline No & $139(38)$ \\
\hline \multicolumn{2}{|c|}{ Neck pain with concomitant headache } \\
\hline Yes & $52(14)$ \\
\hline No & $315(86)$ \\
\hline \multicolumn{2}{|l|}{ How often do you? } \\
\hline \multicolumn{2}{|c|}{ Use CT for new-onset headache (not acute headache) } \\
\hline$\geq 2 / 3$ times & $7(2)$ \\
\hline $1 / 2$ times & $19(5)$ \\
\hline$\leq 1 / 3$ times & $341(93)$ \\
\hline \multicolumn{2}{|c|}{ Use MRI for new-onset headache (not acute headache) } \\
\hline$\geq 2 / 3$ times & $66(18)$ \\
\hline $1 / 2$ times & $56(15)$ \\
\hline$\leq 1 / 3$ times & $245(67)$ \\
\hline \multicolumn{2}{|c|}{ Use CT for long-lasting headaches } \\
\hline$\geq 2 / 3$ times & $17(5)$ \\
\hline $1 / 2$ times & $24(7)$ \\
\hline$\leq 1 / 3$ times & $326(89)$ \\
\hline \multicolumn{2}{|c|}{ Use MRI for long-lasting headaches } \\
\hline$\geq 2 / 3$ times & $103(28)$ \\
\hline $1 / 2$ times & $69(19)$ \\
\hline$\leq 1 / 3$ times & $195(53)$ \\
\hline \multicolumn{2}{|c|}{ Find $C T$ useful in headache investigations } \\
\hline$\geq 2 / 3$ times & $41(11)$ \\
\hline $1 / 2$ times & $36(10)$ \\
\hline$\leq 1 / 3$ times & $290(79)$ \\
\hline \multicolumn{2}{|c|}{ Find MRI useful in headache investigations } \\
\hline$\geq 2 / 3$ times & $153(42)$ \\
\hline $1 / 2$ times & $64(17)$ \\
\hline$\leq 1 / 3$ times & $150(41)$ \\
\hline
\end{tabular}


Table 3 The use of imaging of headache patients among general practitioners. All figures are numbers (\%) (Continued)

\begin{tabular}{lc}
\hline & All $\mathbf{( N = 3 6 7 )}$ \\
\hline Use CT or MRI to alleviate a patient's concerns/anxiety about tumor cerebri or other intracranial pathology \\
$\geq 2 / 3$ times & $72(20)$ \\
$1 / 2$ times & $75(20)$ \\
$\leq 1 / 3$ times & $220(60)$ \\
\hline
\end{tabular}

Gender and specialist status did not influence the ranking of barriers.

The most common reason for referring headache patients was treatment failure/lack of good treatment options in primary care (median 2, IQR $1-2, p<0.001$ compared to all other reasons). Diagnostic uncertainty (median 2, IQR 1-3) was the second most common reason, followed by suspicion of serious underlying cause (median 2, IQR 1-4) and the patient's own wishes/expectations (median 3, IQR 2-4).

\section{Discussion}

A main finding in this nationwide questionnaire-based study among GPs was large differences in the knowledge of management of CTTH and $\mathrm{MOH}$ compared to migraine. These findings and potential gaps are important and should be further explored as headache disorders are one of the main reasons for contact with GP, and the large majority of headache patients are treated in primary care.

\section{Strengths and limitations}

The study sample consisted of 367 GPs recruited from a sample of $130 \mathrm{CME}$ groups, and represents as many as $7.6 \%$ of all GPs in Norway $(N=4787)$ when the survey was conducted. Participants were asked to complete questionnaires individually in a web-based questionnaire;

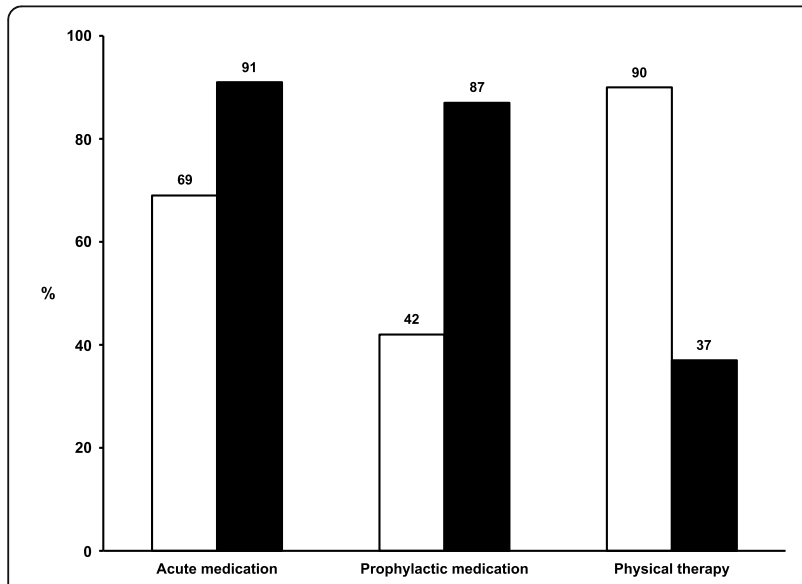

Fig. 3 Percentage of respondents who would use different types of treatments for patients with chronic tension-type headache (white bars) and frequent episodic migraine (1-2 attacks per week) (black bars) thus, cluster-effect based on CME groups is less likely. Together with a high response rate of CME groups (73\%), this should ensure reasonable representativeness and generalizability. However, potential selection bias from those willing to participate will always be a limitation in such studies. The study assesses GPs' views on headache treatment and assessment, which may differ from their actual practice. The study assesses the most common headache disorders (TTH, migraine, $\mathrm{MOH}$ and TACs), but the findings of knowledge and management may not be extrapolated to all other headache disorders. The questionnaire has not been validated, but is based on two similar studies conducted in the US and in Norway $[3,17]$.

\section{Interpretation of results and comparison with other studies}

More than $50 \%$ of the GPs reported headache management to be clinically difficult, which underlines the importance of educating GPs to be comfortable in diagnosing and treating headache disorders $[16,19]$. No diagnostic tests exist for headache disorders, and diagnoses are primarily based on good history taking and clinical examination. All treatment guidelines are based on specific diagnoses, thus it is of uttermost importance to make the correct diagnosis. Although previous studies among GPs have shown that many patients do not receive a specific headache diagnosis, our finding that only $8 \%$ used the diagnostic headache criteria (ICHD-3) on a regular basis was lower than expected [17, 20-23]. Still, this finding is in line with previous studies that have found that many physicians do not use formal diagnostic classifications as they find them impractical for use in daily practice [21, 24]. Headache diaries, which are recommended for diagnosis and follow-up, were used regularly by approximately one in three GPs.

Neuroimaging is not an essential part of headache investigations and should be reserved for those with red flags indicating secondary headaches [12, 25]. Several studies have shown that neuroimaging is routinely ordered despite a typical headache history and normal clinical examination [26, 27]. Almost all the GPs in our study used imaging for headache with focal neurological symptoms, a typical "red flag", which is in line with the guidelines. Also, few GPs used imaging in patients with concomitant neck pain and headache, where imaging is 


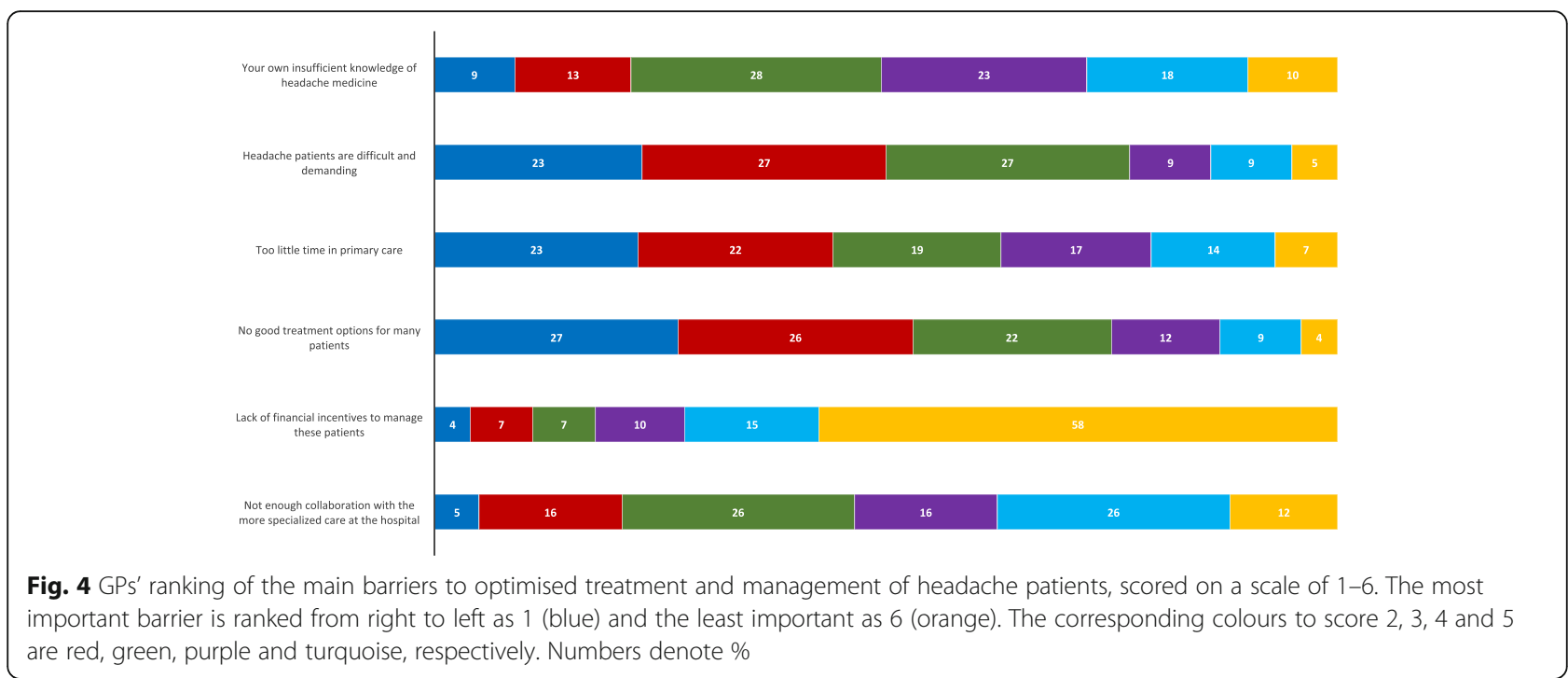

typically of little value. This contrasts with findings from a large multi-national study [28]. Almost two in three GPs used imaging if the patients had concerns and anxiety about brain tumor or other intracranial pathology, suggesting that patient pressure and expectations play a role in decision of whether or not to do neuroimaging. This may be a contributing explanation for the high use of neuroimaging in headache patients. Unnecessary imaging comes with a cost. In addition to the direct costs of the procedure, it may lead to unnecessary fear, repeated investigations, and follow-up of incidental findings. Despite its common use, it is noteworthy that the GPs did not necessarily find imaging very useful.

Effective treatments exist for the most common primary headache disorders, migraine and tension-type headache $[12,29]$. About one in three GPs reported that they had good knowledge about migraine and TTH, and $>96 \%$ reported good or medium knowledge about migraine and TTH. The reported treatment suggestions for migraine were largely in line with this self-reported good knowledge of migraine. The vast majority suggested triptans for acute treatment of migraine and adequate prophylactic medication was suggested for frequent episodic migraine by as many as $87 \%$. Candesartan and beta-blockers are two of the first line choices in most treatment guidelines and were the most commonly suggested prophylactic medications [12-14, 30]. Few GPs suggested topiramate and Botulinum toxin type A. Topiramate has class I evidence and should probably be used more by patients with migraine. The use of Botulinum toxin type A is highly restricted in Norway, and in the study period they could only be prescribed by neurologists to selected chronic migraine patients who had failed $>3$ prophylactic medications. The present study was conducted prior to the introduction of the
CGRP-antibodies in Norway, however, these are now subject to similar limitations and prescription must go through specialists in neurology.

As opposed to the case for migraine treatment, less than half of the respondents suggested prophylactic medication for $\mathrm{CTTH}$, and only $28 \%$ suggested what would be regarded as the first choice in the treatment of CTTH (amitriptyline). Even more worrying, one-third of the participants wrongly stated that the most commonly used headache prophylactics (anti-hypertensives, antiepileptics, and antidepressant drugs) could lead to $\mathrm{MOH}$. This misinterpretation can lead to a worse clinical outcome, and may be one of several explanations for under-use of prophylactics in headache disorders [5, 12, 29, 31]. Most of the respondents knew that simple analgesics and combination analgesics may induce $\mathrm{MOH}$. However, almost 30\% did not know that triptans may induce $\mathrm{MOH}$ and similar to what has been found among primary care physicians in the US and among Norwegian neurology residents, $24 \%$ were unaware that opioids may lead to $\mathrm{MOH}[3,17]$.

GP specialists have a more consistent use and knowledge of headache treatment compared to nonspecialists, probably due to accumulated clinical experience. In line with previous European epidemiological studies simple analgesics were believed to be the most commonly used medication by chronic headache patients [5, 32, 33]. Sixty percent found $\mathrm{MOH}$ to be a clinical challenge among their headache patients. Evidencebased treatment with Brief Intervention for $\mathrm{MOH}$ in primary care does exist and it is encouraging that almost all the GPs approached patients with $\mathrm{MOH}$ with withdrawal and according to the newly published European Academy of Neurology guidelines [34-36]. However, based on the modest knowledge about which 
medications may induce $\mathrm{MOH}$, it may be that many headache patients with $\mathrm{MOH}$ are still not diagnosed and treated adequately, and may remain unrecognized in primary care. $\mathrm{MOH}$ is in principle preventable, thus, identification and information to patients at risk are important [34].

The GPs found "No good treatment options for many patients" to be the main barrier to more optimised headache care. This may be true for certain headache patients, such as patients with frequent or chronic TTH, but at least for migraine, evidence-based treatment options are easily available for most patients in primary care. Headache disorders may be chronic conditions, and patients typically have a need for testing different treatments and long-term follow-up. This may be part of the reason why many GPs find headache patients difficult and demanding, and that they find that the lack of time in general practice contribute to reduced care. Though only $9 \%$ mentioned insufficient knowledge as a main barrier, it is obvious, based on the suboptimal management of CTTH and $\mathrm{MOH}$ in the present study that more knowledge on these entities is needed.

The societal costs of headache are high, increase with severity of symptoms and referral to specialized care [10, 37]. Thus, improved management in primary health care would be of benefit for both patients and society. As there is still a large knowledge gap about how patients are diagnosed and treated in primary care, further studies among GPs may give supplemental information needed to lay the ground for educational efforts such as systematic headache training in CME groups $[16,21$, 38]. Such surveys should be repeated to evaluate time trends and whether specific interventions affect the management and knowledge of headache. In addition, quality indicators of adequate management should be developed and validated specifically for headache in primary care [39]. This may be a first step towards a highquality, predictable management of headache disorders.

\section{Conclusion}

Most of the GPs follow the national recommendations for migraine, but the clinical knowledge of CTTH and $\mathrm{MOH}$ treatment varies. GPs often used MRI in the diagnostic work-up contrary to the recommendations. A more structured headache education for GPs could have direct relevance for better clinical outcomes and reduced costs.

\section{Abbreviations}

GP: General practitioner; CME: Continuous medical education; ICHD3: International Classification of Headache Disorders 3rd edition;

$\mathrm{CTTH}$ : Chronic tension-type headache; $\mathrm{MOH}$ : Medication-overuse headache; TACs: Trigeminal autonomic cephalalgias; NSAIDs: Nonsteroidal antiinflammatory drugs; CGRP: Calcitonin gene-related peptide; GPs: General practitioners; SD: Standard deviations; Cl: Confidence interval;

IQR: Interquartile range.

\section{Supplementary Information}

The online version contains supplementary material available at https://doi. org/10.1186/s10194-021-01350-3.

\section{Additional file 1}

\section{Acknowledgments}

We thank Eira Ebbs, University of Oslo, Norway, for her assistance with translating the questionnaire.

\section{Authors' contributions}

ESK had the original idea for the study and planned the overall design with JCF and CL. ESK prepared the initial draft, and was the main author of the present manuscript. All authors planned the statistics methodology and were involved in all analyses. All authors have read, revised, and approved the final manuscript.

\section{Funding}

This study is supported by grants from the University of Oslo, Akershus University Hospital, and Oslo University Hospital. The funding sources had no role in the design of the study, the collection, analysis, and interpretation of the data, preparation of the manuscript, or the decision to submit the manuscript for publication.

\section{Availability of data and materials}

The authors declare that the data supporting the findings of this study are available within the article.

\section{Declarations}

\section{Ethics approval and consent to participate}

The Regional Committee for Medical Research Ethics reviewed the study and the Norwegian Social Science Data Services approved the study. Informed consent was obtained from all participants.

\section{Consent for publication}

Not applicable.

\section{Competing interests}

$\mathrm{CL}$ has received speaking fees from Abbvie Pharma AS, Novartis AS, Lundbeck AS and Roche AS, Norway, unrelated to the present work CL has also received research sponsorship and payment for advisory board participation from Abbvie Pharma unrelated to the present work. EAT has received speaker honoraria from Allergan, Novartis, Teva, Roche and Lundbeck unrelated to the present work. EAT has received personal fees from global advisory boards from Amgen/Novartis and Eli-Lilly unrelated to the present work. EAT is a Palion Medical and Nordic Brain Tech shareholder; unrelated to the present work. JMH has received speaking fees from Lundbeck, TEVA and Novartis, unrelated to the present work. ESK, KWF, JCF and BSW report no conflicts of interest.

\section{Author details}

${ }^{1}$ Department of General Practice, University of Oslo, PO Box 1130, Blindern, 0318 Oslo, Norway. ${ }^{2}$ Department of Neurology, Akershus University Hospital, Lørenskog, Norway. ${ }^{3}$ Department of Research and Innovation, Division of Clinical Neuroscience, Oslo University Hospital, Oslo, Norway. " Danish Knowledge Centre on Headache Disorders, Rigshospitalet-Glostrup, University, of Copenhagen, Glostrup, Denmark. ${ }^{5}$ Department of Neuromedicine and Movement Science, NTNU Norwegian University of Science and Technology, Trondheim, Norway. ${ }^{6}$ Department of Neurology, National Advisory Unit on Headaches, St. Olavs Hospital, Trondheim, Norway. ${ }^{7}$ Department of Health Management and Health Economics, Institute of Health and Society, University of Oslo, Oslo, Norway. ${ }^{8}$ Health Services Research Unit, Akershus University Hospital, Lørenskog, Norway. ${ }^{9}$ Institute of Clinical Medicine, University of Oslo, Oslo, Norway. ${ }^{10}$ Department of Neurology, Oslo University Hospital, Oslo, Norway. 
Received: 1 October 2021 Accepted: 28 October 2021 Published online: 11 November 2021

\section{References}

1. Ong JJY, Chan YC (2017) Medical undergraduate survey on headache education in Singapore: knowledge, perceptions, and assessment of unmet needs. Headache 57(6):967-978. https://doi.org/10.1111/head.13110

2. World Health Organization (2011) Atlas of headache disorders and resources in the world:2011

3. Kristoffersen ES, Faiz KW, Winsvold BS (2019) Neurology residents' knowledge of the management of headache. Cephalalgia 39(11):1396-1406. https://doi.org/10.1177/0333102419847973

4. Gallagher RM, Alam R, Shah S, Mueller L, Rogers JJ (2005) Headache in medical education: medical schools, neurology and family practice residencies. Headache 45(7):866-873. https://doi.org/10.1111/j.1526-4610.2 005.05155.x

5. Kristoffersen ES, Grande RB, Aaseth K, Lundqvist C, Russell MB (2012) Management of primary chronic headache in the general population: the Akershus study of chronic headache. J Headache Pain 13(2):113-120. https://doi.org/10.1007/s10194-011-0391-8

6. Latinovic R, Gulliford M, Ridsdale L (2006) Headache and migraine in primary care: consultation, prescription, and referral rates in a large population. J Neurol Neurosurg Psychiatry 77(3):385-387

7. Ridsdale L, Clark LV, Dowson AJ, Goldstein LH, Jenkins L, McCrone P, Morgan M, Seed PT (2007) How do patients referred to neurologists for headache differ from those managed in primary care? Br J Gen Pract 57(538):388-395

8. Frese T, Druckrey H, Sandholzer H (2014) Headache in general practice: frequency, management, and results of encounter. Int Scholarly Res Notices 2014:169428. https://doi.org/10.1155/2014/169428

9. Steiner TJ, Antonaci F, Jensen R, Lainez MJ, Lanteri-Minet M, Valade D (2011) Recommendations for headache service organisation and delivery in Europe. J Headache Pain 12(4):419-426. https://doi.org/10.1007/s10194-0110320-x

10. Kristoffersen ES, Stavem K, Lundqvist C, Russell MB (2019) Impact of chronic headache on workdays, unemployment and disutility in the general population. J Epidemiol Community Health 73(4):360-367. https://doi.org/1 0.1136/jech-2018-211127

11. Steiner TJ, Stovner LJ, Vos T, Jensen R, Katsarava Z (2018) Migraine is first cause of disability in under 50s: will health politicians now take notice? J Headache Pain 19(1):17. https://doi.org/10.1186/s10194-018-0846-2

12. Eigenbrodt AK, Ashina H, Khan S, Diener HC, Mitsikostas DD, Sinclair AJ, Pozo-Rosich P, Martelletti P, Ducros A, Lantéri-Minet M, Braschinsky M, Del Rio MS, Daniel O, Özge A, Mammadbayli A, Arons M, Skorobogatykh K, Romanenko V, Terwindt GM, Paemeleire K, Sacco S, Reuter U, Lampl C, Schytz HW, Katsarava Z, Steiner TJ, Ashina M (2021) Diagnosis and management of migraine in ten steps. Nat Rev Neurol 17(8):501-514. https://doi.org/10.1038/s41582-021-00509-5

13. National Advisory Unit on Headache in Norway (2016) Headache diagnosis, examination and treatment. https://stolav.no/fag-og-forskning/ kompetansetjenester-og-sentre/nasjonal-kompetansetjeneste-for-hodepine/ hodepine-diagnose-utredning-og-behandling\#retningslinjer-for-handtering-a v-hodepine

14. Schytz HW, Amin FM, Jensen RH, Carlsen L, Maarbjerg S, Lund N, Aegidius K, Thomsen LL, Bach FW, Beier D, Johansen H, Hansen JM, Kasch H, Munksgaard SB, Poulsen L, Sørensen PS, Schmidt-Hansen PT, Cvetkovic W, Ashina M, Bendtsen L (2021) Reference programme: diagnosis and treatment of headache disorders and facial pain. Danish headache society, 3rd edition, 2020. J Headache Pain 22(1):22

15. Bendtsen L, Sacco S, Ashina M, Mitsikostas D, Ahmed F, Pozo-Rosich P, Martelletti P (2018) Guideline on the use of onabotulinumtoxinA in chronic migraine: a consensus statement from the European headache federation. J Headache Pain 19(1):91. https://doi.org/10.1186/s10194-018-0921-8

16. Minen M, Shome A, Halpern A, Tishler L, Brennan KC, Loder E, Lipton R, Silbersweig D (2016) A migraine management training program for primary care providers: an overview of a survey and pilot study findings, lessons learned, and considerations for further research. Headache 56(4):725-740. https://doi.org/10.1111/head.12803

17. Minen MT, Loder E, Tishler L, Silbersweig D (2016) Migraine diagnosis and treatment: a knowledge and needs assessment among primary care providers. Cephalalgia 36(4):358-370. https://doi.org/10.1177/0333102415593 086

18. The Norwegian Directorate of Health (2019) GP statistics. https://www helsedirektoratet.no/statistikk/fastlegestatistikk\#fastlegestatistikk-2

19. Braschinsky M, Haldre S, Kals M, lofik A, Kivisild A, Korjas J, Koljal S, Katsarava Z, Steiner TJ (2016) Structured education can improve primary-care management of headache: the first empirical evidence, from a controlled interventional study. J Headache Pain 17(1):24. https://doi.org/10.1186/s101 94-016-0613-1

20. Kernick D, Stapley S, Hamilton W (2008) GPs' classification of headache: is primary headache underdiagnosed? Br J Gen Pract 58(547):102-104. https:// doi.org/10.3399/bjgp08X264072

21. Bosner S, Hartel S, Diederich J, Baum E (2014) Diagnosing headache in primary care: a qualitative study of GPs' approaches. Br J Gen Pract 64(626): e532-e537. https://doi.org/10.3399/bjgp14X681325

22. Kernick D, Stapley S, Goadsby PJ, Hamilton W (2008) What happens to newonset headache presented to primary care? A case-cohort study using electronic primary care records. Cephalalgia 28(11):1188-1195. https://doi. org/10.1111/j.1468-2982.2008.01674.x

23. Maizels M (2001) Headache evaluation and treatment by primary care physicians in an emergency department in the era of triptans. Arch Intern Med 161(16):1969-1973. https://doi.org/10.1001/archinte.161.16.1969

24. Farrokhyar F, McHugh K, Irvine EJ (2002) Self-reported awareness and use of the international classification of diseases coding of inflammatory bowel disease services by Ontario physicians. Can J Gastroenterol 16(8):519-526. https://doi.org/10.1155/2002/619574

25. Do TP, Remmers A, Schytz HW, Schankin C, Nelson SE, Obermann M, Hansen JM, Sinclair AJ, Gantenbein AR, Schoonman GG (2019) Red and orange flags for secondary headaches in clinical practice: SNNOOP10 list. Neurology 92(3):134-144. https://doi.org/10.1212/WNL.0000000000006697

26. Callaghan BC, Kerber KA, Pace RJ, Skolarus L, Cooper W, Burke JF (2015) Headache neuroimaging: routine testing when guidelines recommend against them. Cephalalgia 35(13):1144-1152. https://doi.org/10.1177/03331 02415572918

27. Young NP, Elrashidi MY, McKie PM, Ebbert JO (2018) Neuroimaging utilization and findings in headache outpatients: significance of red and yellow flags. Cephalalgia 38(12):1841-1848. https://doi.org/10.1177/03331 02418758282

28. Viana M, Khaliq F, Zecca C, Figuerola MDL, Sances G, Di Piero V, Petolicchio B, Alessiani M, Geppetti P, Lupi C, Benemei S, lannacchero R, Maggioni F, Jurno ME, Odobescu S, Chiriac E, Marfil A, Brighina F, Barrientos Uribe N, Pérez Lago C, Bordini C, Lucchese F, Maffey V, Nappi G, Sandrini G, Tassorelli C (2020) Poor patient awareness and frequent misdiagnosis of migraine: findings from a large transcontinental cohort. Eur J Neurol 27(3):536-541. https://doi.org/10.1111/ene.14098

29. Ashina S, Mitsikostas DD, Lee MJ, Yamani N, Wang SJ, Messina R, Ashina H, Buse DC, Pozo-Rosich P, Jensen RH, Diener HC, Lipton RB (2021) Tensiontype headache. Nat Rev Dis Primers 7(1):24. https://doi.org/10.1038/s41572021-00257-2

30. Kouremenos E, Arvaniti C, Constantinidis TS, Giannouli E, Fakas N, Kalamatas T, Kararizou E, Naoumis D, Mitsikostas DD (2019) Consensus of the Hellenic headache society on the diagnosis and treatment of migraine. J Headache Pain 20(1):113. https://doi.org/10.1186/s10194-019-1060-6

31. Diamond S, Bigal ME, Silberstein S, Loder E, Reed M, Lipton RB (2007) Patterns of diagnosis and acute and preventive treatment for migraine in the United States: results from the American Migraine Prevalence and Prevention study. Headache 47(3):355-363. https://doi.org/10.1111/j.1526-4 610.2006.00631x

32. Westergaard ML, Glumer C, Hansen EH, Jensen RH (2014) Prevalence of chronic headache with and without medication overuse: associations with socioeconomic position and physical and mental health status. Pain 155(10): 2005-2013. https://doi.org/10.1016/j.pain.2014.07.002

33. Lantéri-Minet M, Auray J-P, El Hasnaoui A, Dartigues J-F, Duru G, Henry P, Lucas C, Pradalier A, Chazot G, Gaudin A-F (2003) Prevalence and description of chronic daily headache in the general population in France. Pain 102(1-2):143-149. https://doi.org/10.1016/s0304-3959(02)00348-2

34. Diener HC, Antonaci F, Braschinsky M, Evers S, Jensen R, Lainez M, Kristoffersen ES, Tassorelli C, Ryliskiene K, Petersen JA (2020) European academy of neurology guideline on the management of medicationoveruse headache. Eur J Neurol 27(7):1102-1116. https://doi.org/10.1111/ ene. 14268 
35. Frich JC, Kristoffersen ES, Lundqvist C (2014) GPs' experiences with brief intervention for medication-overuse headache: a qualitative study in general practice. Br J Gen Pract 64(626):e525-e531. https://doi.org/10.3399/ bjgp14X681313

36. Kristoffersen ES, Straand J, Vetvik KG, Benth JS, Russell MB, Lundqvist C (2015) Brief intervention for medication-overuse headache in primary care. The BIMOH study: a double-blind pragmatic cluster randomised parallel controlled trial. J Neurol Neurosurg Psychiatry 86(5):505-512. https://doi. org/10.1136/jnnp-2014-308548

37. Osumili B, McCrone P, Cousins S, Ridsdale L (2018) The economic cost of patients with migraine headache referred to specialist clinics. Headache 58(2):287-294. https://doi.org/10.1111/head.13210

38. Braschinsky M, Haldre S, Kals M, Arge M, Saar B, Niibek M, Katsarava Z, Steiner TJ (2018) Structured education to improve primary-care management of headache: how long do the benefits last? A follow-up observational study. Eur J Neurol 25(3):497-502. https://doi.org/10.1111/ ene.13524

39. Lenz B, Katsarava Z, Gil-Gouveia R, Karelis G, Kaynarkaya B, Meksa L, Oliveira E, Palavra F, Rosendo I, Sahin M, Silva B, Uludüz D, Ural YZ, Varsberga-Apsite I, Zengin ST, Zvaune L, Steiner TJ (2021) Headache service quality evaluation: implementation of quality indicators in primary care in Europe. J Headache Pain 22(1):33. https://doi.org/10.1186/s10194-021-01236-4

\section{Publisher's Note}

Springer Nature remains neutral with regard to jurisdictional claims in published maps and institutional affiliations.

Ready to submit your research? Choose BMC and benefit from:

- fast, convenient online submission

- thorough peer review by experienced researchers in your field

- rapid publication on acceptance

- support for research data, including large and complex data types

- gold Open Access which fosters wider collaboration and increased citations

- maximum visibility for your research: over $100 \mathrm{M}$ website views per year

At $\mathrm{BMC}$, research is always in progress.

Learn more biomedcentral.com/submissions 